\title{
A MATHEMATICAL THEORY OF OPTIMAL ECONOMIC DEVELOPMENT ${ }^{1}$
}

\author{
BY DAVID GALE
}

1. Introduction. The general aim of this article is two-fold; first, to show by means of examples the kinds of mathematical problems which arise from economic situations, and second, to illustrate the principal methods by which these problems can be attacked. I have endeavored to present this material in a manner appropriate both for the general mathematician and the mathematically oriented economist. For the former whose interest is probably mainly one of curiosity, I have tried to convey the general flavor of the subject-a sort of answer to the question "what is mathematical economics?" For the latter I hope that these examples will provide useful illustrations of the power of modern optimization theory in attacking economic problems.

For achieving the above purposes the subject of economic development seems a particularly suitable vehicle. The problems are easily described and can be formulated mathematically in a variety of ways. An economy consists of various "goods" which can be used for two purposes: they can either be "invested" in which case they produce more of themselves, or they can be "consumed" in which case they provide satisfaction or "utility." The entire structure of the economy is given by specifying how these two operations can be carried on. The specification of how goods can be transformed into each other is called the technology of the model and the specification of how goods are transformed to satisfaction is called the utility function. Given this structure and some initial bundle of goods, the problem of optimal development is to decide at each point of time how much to invest and how much to consume in order to maximize utility summed over time in some suitable way.

Having arrived at a definition of an optimal development program one proceeds to ask the usual questions. Are there any such programs?

An address delivered to a joint meeting of the American Mathematical Society and the Econometric Society in Toronto on August 30,1967, by invitation of the Committee to Select Hour Speakers for Summer and Annual Meetings; received by the editors September 27, 1967.

${ }^{1}$ Research reported on here was partially supported by a National Science Foundation Grant GP-4593 and by the Office of Naval Research under contract Nonr 222(83). 
If so, are they unique? What are their qualitative properties, e.g. are they monotonic, with utility getting greater each day, or are there situations in which things have to get worse before they can get better and so on? The bulk of this exposition is concerned with such questions, but the emphasis will be less on the specific answers obtained than on the technique used in obtaining them. The technique in question is the method of price systems, and it is the exploitation of this concept which constitutes the heart of the present exposition. It may be helpful in conclusion to describe roughly what this method is.

Note, first, that in our description of an economy, the notion of money, prices, profits, interest rates and the like do not appear. The formulation is entirely in terms of "real" economic quantities, namely, goods and utility. The remarkable and somewhat surprising fact is, however, that in order to answer questions dealing only with real quantities it has been found necessary to introduce the ideas of money, prices and profits. As an example, the only way I know for proving the existence of certain classes of optimal programs involves showing first that there exist programs which are profit maximizing under a suitable price system and then showing that such profit maximizing programs must be optimal. I emphasize again that the prices are not part of the model to begin with but are brought in for purposes of mathematical analysis. Of course, the theorems about prices which one proves are of considerable economic interest in themselves, for they show, roughly speaking, that if prices are chosen correctly then optimal welfare is achieved by having each productive facility maximize its own profits. This idea, I hasten to add, is far from new. On the contrary, the proposition that proper pricing can bring about optimal allocation of resources may well be the central idea in all of economic theory. Modern global optimization theory, developed largely since the end of World War II, has only served to emphasize and reenforce this proposition, sometimes in quite spectacular ways. The sections to follow are intended to illustrate this phenomenon in the context of economic development.

2. Goods, utility, technologies and programs. We consider an economy in which there are $m$ goods. A nonnegative $m$-vector $x$ $=\left(x_{1}, \cdots, x_{m}\right)$ is called a goods bundle in which $x_{i}$ is the amount of the $i$ th good in the bundle $x$.

Goods can be used for two purposes. They can either be consumed thus providing satisfaction or utility to consumers, or they can be invested, in which case they produce more of themselves in some specified way. This is made precise as follows: 
There is a utility function defined on all goods bundles, which may vary with time. We write $u_{t}(x)$ and interpret this as the satisfaction to society of consuming the bundle $x$ at time $t$. It is assumed in this model that the bundle $x$ disappears in the act of being consumed. There is some difficulty with this formulation. Many things people do for satisfaction do not seem to use anything up. Looking at pictures in a museum, for example, does not cause the pictures to disappear. The goods that get consumed in this case are not the pictures but the viewer's leisure time which in this formulation must itself be considered a good. This seems somewhat awkward and we will later propose an alternative formulation which gets around this problem.

A technology $J$ is a set of pairs of goods bundles $(x, y)$. The interpretation is that starting with the bundle $x$ it is possible to produce from it the bundle $y$ one time period later. We will refer to the pair $(x, y)$ as a productive activity and call $x$ and $y$ the input and output of the activity.

In considering the question of development, it will be assumed that at the present time $(t=0)$ there is on hand a bundle $s$ called the initial stocks. It is now quite clear what one should mean by a development program.

Definition 1. A program with initial stocks $s$ is a sequence of activities $\left\langle x_{t}, y_{t}\right\rangle, t=0,1, \cdots$ (finite or infinite), where

$$
\left(x_{t}, y_{t}\right) \in J, \quad x_{0} \leqq s, \quad x_{t} \leqq y_{t-1}, \quad t \geqq 1 .
$$

The consumption $c_{t}$ in period $t$ is defined by

$$
c_{0}=s-x_{0}, \quad c_{t}=y_{t-1}-x_{t} .
$$

The sequence $\left\langle u_{t}\left(c_{t}\right)\right\rangle$ is called the utility sequence of the program $\left\langle x_{t}, x_{t}\right\rangle$.

Conditions (1.1) state that input in period $t$ cannot exceed output of period $t-1$. Condition (2.1) defines the difference of these to be consumption. (It may be that some of the goods in $c_{t}$ are not consumed in the literal sense but merely thrown away, but there is no reason why one cannot attach utility (or disutility) to this act as well as that of, say, eating.)

If a program is finite, say, $t=0,1, \cdots, T$, then the bundle $y_{T}$ is called the bundle of final stocks. A $T$-period program with final stocks $y_{T}=s^{\prime}$ will be called a program from $s$ to $s^{\prime}$. The optimality concept now enters in a natural way.

Definition 2. A $T$-period program from $s$ to $s^{\prime}$ is called optimal if it maximizes $\sum_{t=0}^{T} u_{t}\left(c_{t}\right)$ among all such programs. 
Although the main subjects in development theory are the infinite programs it is necessary first to look briefly at finite programs which we do in the next section.

3. Prices and competitive programs. We now introduce the main concept of this paper.

Definition 3. A program $\left\langle x_{t}, y_{t}\right\rangle$ is called competitive if there exists a sequence of $m$-vectors $p_{t}$, called prices, such that for all $t$

(A) $p_{t+1} y-p_{t} x \leqq p_{t+1} y_{t}-p_{t} x_{t}$ for all $(x, y)$ in $J$,

(B) $u_{t}(c)-p_{t} c \leqq u_{t}\left(c_{t}\right)-p_{t} c_{t}$ for all $c \geqq 0$.

The economic interpretation of these conditions is important. The vector $p_{t}$ gives the prices of the $m$ goods in period $t$. Thus in (A) the term $p_{t} x$ is the cost of the input $x$ in period $t$ and $p_{t+1} y$ is the value of the output $y$ in period $t+1$. Their difference is, of course, the profit obtained from the activity $(x, y)$ and condition (A) states that the activity $\left(x_{t}, y_{t}\right)$ maximizes profits at prices $p_{t}$ and $p_{t+1}$ among all possible pairs in J. Briefly, producers maximize profits.

Condition (B) is the consumption condition and it says that consumers maximize utility, subject to their budgetary constraints, the constraint being that they may spend no more than the amount $p_{t} c_{t}$. This follows because if there were a bundle $c^{\prime}$ which cost at most $p_{t} c_{t}$ and gave a higher value of $u$ we would have $u\left(c^{\prime}\right)-p_{t} c^{\prime}>u\left(c_{t}\right)-p_{t} c_{t}$ contrary to (B). Further it is reasonable to limit consumption expenditure to $p_{t} c_{t}$ for by definition $c_{t}=y_{t-1}-x_{t}$, so $p_{t} c_{t}=p_{t} y_{t-1}-p_{t} x_{t}$. The term on the right represents the value of yesterday's output minus the value of what is to be invested today and the difference is what is available for today's consumption.

The following simple result is the starting point for our theory.

THEOREM 1. If a T-period program from $s$ to $s^{\prime}$ is competitive, it is optimal.

PRoof. Let $\left\langle x_{t}^{\prime}, y_{t}^{\prime}\right\rangle$ be any other program from $s$ to $s^{\prime}$ and let $\left\langle p_{t}\right\rangle$ be the competitive prices. Then from (B)

$$
\begin{gathered}
u\left(c_{0}^{\prime}\right)-u\left(c_{0}\right) \leqq p_{0}\left(c_{0}^{\prime}-c_{0}\right)=p_{0}\left(s-x_{0}^{\prime}\right)-p\left(s-x_{0}\right)=p_{0} x_{0}-p_{0} x_{0}^{\prime}, \\
u\left(c_{t}^{\prime}\right)-u\left(c_{t}\right) \leqq p_{t}\left(c_{t}^{\prime}-c_{t}\right)=p_{t}\left(y_{t-1}^{\prime}-x_{t}^{\prime}\right)-p_{t}\left(y_{t-1}-x_{t}\right) \\
\quad \text { for } 1 \leqq t \leqq T, \\
0=p_{T+1}\left(s^{\prime}-s^{\prime}\right)=p_{T+1} y_{T}^{\prime}-p_{T+1} y_{T} .
\end{gathered}
$$

Summing from 0 to $T$ and rearranging gives 


$$
\sum_{t=0}^{T} u\left(c_{t}^{\prime}\right)-\sum_{t=0}^{T} u\left(c_{t}\right) \leqq \sum_{t=0}^{T}\left[\left(p_{t+1} y_{t}^{\prime}-p_{t} x_{t}^{\prime}\right)-\left(p_{t+1} y_{t}-p_{t} x_{t}\right)\right],
$$

but from (A) every term on the right is nonpositive, hence

$$
\sum_{t=0}^{T} u\left(c_{t}^{\prime}\right) \leqq \sum_{t=0}^{T} u\left(c_{t}\right)
$$

and $\left\langle x_{t}, y_{t}\right\rangle$ is optimal.

The next objective is to obtain a converse of this theorem. For this purpose some mathematical preliminaries are needed.

4. The Kuhn-Tucker Theorem and existence of competitive programs. From now on we will assume that the following two conditions are satisfied.

I. The technology $J$ is a convex subset of $2 m$-space.

II. The utility $u_{t}$ is continuous concave in $c$ for all $t$.

These conditions are in many cases economically reasonable. For the technology if $(x, y)$ and $\left(x^{\prime}, y^{\prime}\right)$ represent feasible activities then if one could carry them both on at the same time one would also have the activity $\left(x+x^{\prime}, y+y^{\prime}\right)$. However, these activities might require certain resources which were only available in limited amounts (e.g. labor). Assuming these resources were "infinitely divisible" one could allocate some fraction $\lambda$ of them to the first activity and $(1-\lambda)$ to the second thus obtaining the activity $\left(\lambda x+(1-\lambda) x^{\prime}, \lambda y+(1-\lambda) y^{\prime}\right)$. As for the utility function, it is at least from a realistic point of view somewhat arbitrary to begin with. The assumption of concavity reflects first the notion of diminishing returns-consuming 100 potatoes is not 100 times as enjoyable as consuming a single potato-and second the idea that diversity is desirable; if people on the average are indifferent between apples and oranges then having some of each is at least as desirable as having all of either one. (This applies only to the whole society. An individual might prefer either an apple or an orange to one half an apple and one half an orange.)

Assumptions I and II are needed so that one can make use of the Kuhn-Tucker Theorem which is not only the key mathematical result needed for the present theory but quite likely the most important single result in modern optimization theory. We shall give a version here which is most convenient for the present application. The theorem is concerned with maximizing a concave function $u$ over a convex set $X$ in $n$-space where $x$ in $X$ is also required to satisfy a set of linear equations, $A x=b$, where $A$ is an $m \times n$ matrix and $b$ is an $n$ vector. 
Kuhn-Tucker Theorem. Suppose $\bar{x}$ in $X$ maximizes $u(x)$ subject to the constraints $A x=b$. Suppose also

(4.1) The set of solutions of $A x=b$ meets the interior of $X$.

Then there is an m-vector $p$ such that the function $u(x)-p A x$ achieves its maximum at $\bar{x}$.

This theorem looks very much like the ordinary theorems about Lagrange multipliers, for the components of $p$ are precisely such multipliers and the function $u(x)-p A x$ is the usual Lagrangian. There are two important differences however.

(I) The usual theory only refers to interior maxima while this theorem is equally valid for maxima which occur on the boundary of $X$ which will of ten be the case in applications.

(II) In the usual Lagrange theory one can only say that if $\bar{x}$ satisfies the constrained maximum problem then the Lagrangian has a critical point at $\bar{x}$. Indeed, the significance of the Kuhn-Tucker Theorem is that it transforms a constrained maximum problem for the function $u(x)$ into an unconstrained maximum problem for the function $u(x)-p A x$.

The proof of the theorem is quite simple depending only on the well-known fact that a concave function $\phi$ on an open convex set $B$ is the minimum of all linear functions $p$ on $B$ such that $p \geqq \phi$. Thus, for any $b$ in $B$ there exists a vector $p$ such that $p \cdot\left(b^{\prime}-b\right) \geqq \phi\left(b^{\prime}\right)-\phi(b)$. We call $p$ a support of $u$ at the point $b$. The interested reader can construct his own proof, as follows: First, let $B$ be the set of all vectors $b^{\prime}$ such that the set $A x=b^{\prime}$ satisfies (4), and show that $B$ is open and convex. Then let $\phi\left(b^{\prime}\right)=\max _{A x=b^{\prime}} u(x)$ and show that $\phi\left(b^{\prime}\right)$ is concave. Finally, let $p$ be a support of $\phi$ at $b$. Then for any $x^{\prime}$ in $X$ letting $A x^{\prime}=b^{\prime}$ we get

$u\left(x^{\prime}\right)-p A x^{\prime}=u\left(x^{\prime}\right)-p b^{\prime} \leqq \phi\left(b^{\prime}\right)-p b^{\prime} \leqq \phi(b)-p b=u(\bar{x})-p A \bar{x}$

which is the conclusion of the Kuhn-Tucker Theorem.

Condition (4.1) is not necessary. An alternative condition which is often useful is that $X$ be a polyhedral set. The proof in this case is a little more difficult. Some condition, however, on the set $X$ is needed as the following example shows.

Let $X=\left\{\left(x_{1}, x_{2}\right) \mid x_{2} \geqq x_{1}^{2}\right\}, u\left(x_{1}, x_{2}\right)=x_{1}$ and let us try to maximize $u$ subject to the condition $x_{2}=0$. Obviously the solution must be $\bar{x}=(0,0)$. However, the Lagrangian $L(x)$ is given by $L(x)=x_{1}-p x_{2}$ and for $p \leqq 0, L$ has no maximum, while for $p>0, L$ attains a maximum of $1 / 4 p$ at $x=\left(1 / 2 p, 1 / 4 p^{2}\right)$. 
There are other conditions on $X$ which assure the validity of the Kuhn-Tucker Theorem, but this is a somewhat technical matter which we will not get into here. It will be assumed for our applications that the technology $I$ satisfies these very mild restrictions (for details see [1]) so that the Kuhn-Tucker Theorem is applicable. Returning now to the development problem we have

TheOREM 2. If $\left\langle\bar{x}_{t}, \bar{y}_{t}\right\rangle$ is an optimal $T$-period program from $s$ to $s^{\prime}$ then it is competitive.

Proof. By definition, $\left\langle\bar{x}_{t}, \bar{y}_{t}\right\rangle$ maximizes $\sum_{t=0}^{T} u_{t}\left(c_{t}\right)$ subject to the constraints

$$
x_{0}+c_{0}=s, \quad x_{t}+c_{t}=y_{t-1}, \quad(t=1, \cdots, T), \quad s^{\prime}=y_{T}
$$

where $\left(x_{t}, y_{t}\right)$ is in $J$ and $c_{t}$ is nonnegative.

From the Kuhn-Tucker Theorem there exist numbers $p_{t}$, $t=0, \cdots, T+1$ such that

$$
\sum_{t=0}^{T} u\left(c_{t}\right)-p_{0}\left(x_{0}+c_{0}\right)-\sum_{t=1}^{T} p_{t}\left(x_{t}+c_{t}-y_{t-1}\right)+p_{T+1} y_{T}
$$

is maximized at $\left\langle\bar{x}_{t}, \bar{y}_{t}, \bar{c}_{t}\right\rangle$. But rearranging (4.2) gives

$$
\sum_{t=0}^{T}\left[\left(u_{t}\left(c_{t}\right)-p_{t} c_{t}\right)+\left(p_{t+1} y_{t}-p_{t} x_{t}\right)\right]
$$

and the $2 T$ terms in (4.3) are independent, so the sum will be a maximum if and only if each term is a maximum, i.e.

$$
\begin{aligned}
& p_{t+1} y_{t}-p_{t} x_{t} \text { is maximized at }\left(\bar{x}_{t}, \bar{y}_{t}\right), \text { and } \\
& u\left(c_{t}\right)-p_{t} c_{t} \text { is maximized at } \bar{c}_{t} .
\end{aligned}
$$

But these are precisely the competitive conditions.

Having established the equivalence of optimal and competitive programs we shall devote the rest of this paper to illustrating how the competitive conditions can be used to analyze properties of development programs.

5. A model with a single good. For purposes of economic analysis it is sometimes useful to construct highly idealized economic models, one might call them animated cartoons of a real economy, which illustrate some particular aspect of an economy in order to gain rough qualitative information on how a real economy might behave. In this section we shall look briefly at such a model. It will involve only one good which can either be consumed to gain utility, or invested in 
which case investing the input $x$ in period $t$ gives rise to an output $f(x)$ in period $t+1$. The function $f$, called the production function, is assumed to be concave. The technology in this case is given by

$$
J=\{(x, y) \mid y \leqq f(x)\}
$$

and since $f$ is concave, $J$ will be convex.

We further assume that the model is productive, that is, increasing the inputs by an amount $h$ increases outputs by more than $h$, so that

$$
f(x+h)-f(x)>h \quad \text { for all } x \geqq 0, \quad h>0 .
$$

Finally we shall assume that the utility function $u$ is independent of time and an increasing function of $c$. This means that any optimal program $\left\langle x_{t}, y_{t}\right\rangle$ must be of the form $\left\langle x_{t}, f\left(x_{t}\right)\right\rangle$ since one will always produce the maximum output possible from any given input so that $c_{t}=f\left(x_{t-1}\right)-x_{t}$. We now give a theorem on the qualitative nature of optimal programs whose statement does not involve the notion of prices but whose proof seems to require their use.

THEOREM 3. Let $\left\langle x_{i}, f\left(x_{t}\right)\right\rangle$ be an optimal T-period program from $s$ to $s^{\prime}$. Then

(a) Consumption $c_{t}$ and utility $u\left(c_{t}\right)$ are nondecreasing functions of $t$.

(b) Inputs $x_{t}$ are increasing up to some time to and decreasing thereafter.

Proof. Since $\left\langle x_{t}, f\left(x_{t}\right)\right\rangle$ is optimal it is competitive, hence there exist prices $p_{t}$ such that from (A)

$$
p_{t+1} f(x)-p_{t} x \leqq p_{t+1} f\left(x_{t}\right)-p_{t} x_{t} \quad \text { for all } x \geqq 0 .
$$

In order to prove (a) and (b) we must first prove

(c) The prices $p_{t}$ are strictly decreasing in $t$.

This follows from (5.2) for we have

$$
p_{t} / p_{t+1} \geqq\left(f(x)-f\left(x_{t}\right)\right) /\left(x-x_{t}\right) \quad \text { for } x>x_{t},
$$

but from (5.1) the term on the right exceeds 1.

Next from competitive condition (B)

$$
\begin{aligned}
& u\left(c_{t}\right)-u\left(c_{t+1}\right) \geqq p_{t}\left(c_{t}-c_{t+1}\right), \\
& u\left(c_{t+1}\right)-u\left(c_{t}\right) \geqq p_{t+1}\left(c_{t+1}-c_{t}\right)
\end{aligned}
$$

and adding gives $\left(p_{t+1}-p_{t}\right)\left(c_{t+1}-c_{t}\right) \leqq 0$ hence since $p_{t+1}<p_{t}$ it follows that $c_{t+1} \geqq c_{t}$ which proves (a).

To prove (b) it suffices to show that if $x_{t}<x_{t-1}$ then $x_{t+1}<x_{t}$. Now since $f$ is increasing we have $f\left(x_{t}\right)<f\left(x_{t-1}\right)$, and from (a) $-c_{t+1} \leqq-c_{t}$, and adding these inequalities gives 


$$
x_{t+1}=f\left(x_{t}\right)-c_{t+1}<f\left(x_{t-1}\right)-c_{t}=x_{t},
$$

as asserted.

6. Infinite programs. Finite programs as discussed in the preceding section are not of great interest in economic development, for one is not usually given the desired final stocks $s^{\prime}$. On the contrary, the central problem in development theory is to decide on the best rate for building up stocks, the so-called capital accumulation problem. To attack this problem one must consider infinite programs, and the first problem is to define a suitable notion of optimality. It obviously will not do to simply maximize the infinite series $\sum_{t=0}^{\infty} u_{t}\left(c_{t}\right)$ since this series will in general diverge. There does turn out, however, to be a natural notion of optimality based on the following partial ordering of programs.

Definition. The program $\left\langle x_{t}, y_{t}\right\rangle$ will be said to overtake the program $\left\langle x_{t}^{\prime}, y_{t}^{\prime}\right\rangle$ if there exists a time $T$ such that

$$
\sum_{t=0}^{T^{\prime}} u\left(c_{t}\right)>\sum_{t=0}^{T^{\prime}} u\left(c_{t}^{\prime}\right) \quad \text { for all } T^{\prime} \geqq T
$$

The program $\left\langle x_{t}, y_{t}\right\rangle$ catches up to $\left\langle x_{t}^{\prime}, y_{t}^{\prime}\right\rangle$ (at infinity) if

$$
\underset{T \rightarrow \infty}{\liminf } \sum_{t=0}^{T}\left(u\left(c_{t}^{\prime}\right)-u\left(c_{t}\right)\right) \geqq 0 .
$$

A program is called optimal (strongly optimal) if it catches up to (overtakes) every other program.

Note that this definition includes as a special case the situation in which the series $\sum u_{t}\left(c_{t}\right)$ does converge (as may occur, for instance, if future utilities are suitably discounted). However, as we shall see, the definition applies as well to situations in which the series definitely does not converge. Of course, for a given technology and utility function it may happen that there are no optimal programs. Here is a simple example for the single good model.

Suppose $u(c)=c$, and from $x$ units of goods we can produce $2 x$ units. The claim is that there can be no optimal program, for let $\left\langle x_{t}, 2 x_{t}\right\rangle$ be any program and suppose $c_{T}=2 x_{T-1}-x_{T}>0$ for some $T$. We construct the overtaking program $\left\langle x_{t}^{\prime}, 2 x_{t}^{\prime}\right\rangle$ by letting it agree with $\left\langle x_{t}, 2 x_{t}\right\rangle$ except for $t=T$ and $T+1$ where we define

$$
c_{T}^{\prime}=0 \quad \text { and } \quad x_{T}^{\prime}=2 x_{T-1}, \quad c_{T+1}^{\prime}=2 x_{T}^{\prime}-x_{T+1} .
$$

Then $c_{T}^{\prime}+c_{T+1}^{\prime}=4 x_{T-1}-x_{T+1}$ while 


$$
c_{T}+c_{T+1}=2 x_{T-1}-x_{T}+2 x_{T}-x_{T+1}=2 x_{T-1}+x_{T}-x_{T+1},
$$

and subtracting gives

$$
c_{T}^{\prime}+c_{T+1}^{\prime}-c_{T}-c_{T+1}=2 x_{T-1}-x_{T}=c_{T}>0,
$$

hence $\left\langle x_{t}^{\prime}, y_{t}^{\prime}\right\rangle$ overtakes $\left\langle x_{t}, y_{t}\right\rangle$.

The above is a very special case of a general result which can be obtained using prices. We ask the reader to accept the fact that infinite optimal programs are competitive. This is proved by a passage to the limit from the finite case but involves a rather delicate point of showing that the prices one gets are uniformly bounded.

THEOREM 4. In the one good model if $f$ is productive and $u$ is unbounded then no optimal program exists.

Proof. Suppose $\left\langle x_{t}, f\left(x_{t}\right)\right\rangle$ was optimal, hence competitive. Then from condition (B),

$$
\begin{gathered}
u\left(c_{1}\right)-u\left(c_{0}\right) \leqq p_{0}\left(c_{1}-c_{0}\right)=p_{0}\left(f\left(x_{0}\right)-x_{1}\right)-p_{0}\left(s-x_{0}\right), \\
u\left(c_{t+1}\right)-u\left(c_{t}\right) \leqq p_{t}\left(c_{t+1}-c_{t}\right)=p_{t}\left(f\left(x_{t}\right)-x_{t+1}\right)-p_{t}\left(f\left(x_{t-1}\right)-x_{t}\right) .
\end{gathered}
$$

Summing from $t=0$ to $T$ gives

$$
\begin{aligned}
u\left(c_{T+1}\right)-u\left(c_{0}\right) \leqq & p_{T}\left(x_{T}-x_{T+1}\right) \\
& \left.+\sum_{t=1}^{T}\left[p_{t} f\left(x_{t}\right)-p_{t-1} x_{t}\right)-\left(p_{t} f\left(x_{t-1}\right)-p_{t-1} x_{t-1}\right)\right] \\
& +p_{0}\left(f\left(x_{0}\right)-s\right) .
\end{aligned}
$$

Now if the function $u$ is unbounded then in any optimal program, clearly, $\left\langle c_{t}\right\rangle$ is unbounded, hence $\left\langle x_{t}\right\rangle$ is unbounded and hence, from Theorem $3,\left\langle x_{t}\right\rangle$ is monotone increasing. This means the first term on the right-hand side of (6.1) is negative. Further, by condition (A) all terms in the summation are negative so (6.1) becomes $u\left(c_{T+1}\right)$ $\leqq u\left(c_{0}\right)+p_{0}\left(f\left(x_{0}\right)-s\right)$, but this means that $u\left(c_{t}\right)$ remains bounded, as asserted.

It is interesting to note that the converse of Theorem 4 is true. If $u$ is bounded then an optimal program does exist. The proof, which is somewhat lengthy, proceeds by first showing that an infinite competitive program exists which has a certain additional property called efficiency, and from this it follows that the program is optimal. The interested reader is referred to [2]. Thus, boundedness of $u$ is seen to be necessary and sufficient for the existence of optimal programs (this was first proved by McFadden for the case when $f$ is a linear function [3]). We shall return to the general existence question later. 
7. Multi-commodity models. The von Neumann technology. We turn now to models with many goods. For this purpose a somewhat different formulation based on some work of von Neumann [4] is convenient. The model involves, first of all, a finite set of $n$ activities, represented by the input-output pairs $\left(a_{j}, b_{j}\right), j=1, \cdots, n$. Each activity can be operated at various levels; thus if $\left(a_{j}, b_{j}\right)$ is an activity and $\nu_{j}$ is a nonnegative number then $\left(\nu_{j} a_{j}, \nu_{j} b_{j}\right)$ is also an activity. Further, the different activities may be operated simultaneously. It is not hard to see now that if $A$ and $B$ are matrices whose $j$ th columns are $a_{j}$ and $b_{j}$, respectively, then for any nonnegative vector $v$ the pair $(A v, B v)$ is an activity. The vector $v$ is called the vector of activity levels or more simply the activity vector. To complete the description of the technology we must restrict the set of possible vectors $v$, for it is obvious that in any real technology it will not be possible to operate all activities at once at arbitrarily high levels due to the scarcity of primary resources of which labor of various kinds is the most important. We assume therefore that the vectors $v$ are restricted to lie in some bounded convex polyhedral set $V$ containing the origin. Given the $m \times n$ matrices $A$ and $B$ and the set $V$ in $n$-space, the technology $J$ is then given by

$$
\boldsymbol{J}=\{(A v, B v) \mid v \in V\} .
$$

In general the set $V$ will vary with time, since population, and therefore the labor force, is generally changing. However, in the case in which all types of labor grow at the same rate, one can transform the development problem into one in which the labor force remains constant. In what follows we shall confine ourselves to this case.

Definition. A program with initial stocks $s$ is a sequence of activity vectors $\left\langle v_{t}\right\rangle$ from $V$ such that

$$
A v_{0}=s, \quad A v_{t}=B v_{t-1} .
$$

Thus, outputs of period $t-1$ are inputs of period $t$. This does not mean that one must actually use all available outputs at all times. An output may simply be disposed of or stored from one period to the next, or allowed to deteriorate, for each of these possibilities may be accounted for by introducing suitable activities in the $A$ and $B$ matrices.

We now bring in the utility function, and at this point we introduce a mild innovation. Instead of defining the utility on goods bundles, we define it as a function $u_{t}$ on activity vectors $v$. Thus, $u_{t}(v)$ is the satisfaction to society from engaging in activities at levels $v$ at time $t$. 
We believe this is really an improvement over the usual formulation. It is not, after all, the piece of cake that produces satisfaction, but the act of consuming it. Likewise there are certain activities, digging ditches for example, which produce dissatisfaction. Finally, the problem previously mentioned of attaching utility to looking at pictures in a museum is easily solved by placing the utility on the viewing activity rather than the pictures.

Definition. A utility function $u_{t}$ is a real valued function on the set of nonnegative $n$-vectors $v$. The utility sequence corresponding to the program $\left\langle v_{t}\right\rangle$ is the sequence $\left\langle u_{t}\left(v_{t}\right)\right\rangle$.

In the new formulation the concept of optimal infinite program remains unchanged. We require that the sequence $\left\langle u_{t}\left(v_{t}\right)\right\rangle$ catch up to or overtake all others. The notion of competitive program becomes even simpler than before, although its economic interpretation is not quite so transparent.

Definition. The program $\left\langle v_{t}\right\rangle$ is competitive if there exist prices $p_{t}$ such that for each $t$

$$
u_{t}(v)+\left(p_{t+1} B-p_{t} A\right) v \leqq u_{t}\left(v_{t}\right)+\left(p_{t+1} B-p_{t} A\right) v_{t}
$$

for all $v$ in $V$.

The condition states that at each time $t, v$ is chosen so as to maximize the sum of profit and utility.

It is now an easy matter to verify that if $\left\langle v_{t}\right\rangle$ is a competitive $T$-period program from $s$ to $s^{\prime}$ then it is optimal. Using the KuhnTucker Theorem one just as easily proves conversely that if $u$ is concave then any optimal $T$-period program is competitive. ${ }^{2}$ Assuming these verifications have been performed we turn our attention to infinite programs.

8. Stationary programs and asymptotic properties of good programs. In this section we will consider only the case where $u$ is independent of the time. We introduce the principal notion of the section.

Definition. A program $\left\langle v_{t}\right\rangle$ is called stationary if $v_{t}=v_{0}$ for all $t$.

From the definition of a program it follows that $v_{0}$ must satisfy

$$
(B-A) v=0 \text {, }
$$

and in fact stationary programs correspond exactly to vectors $v$ in $V$ which satisfy (8.1).

An optimal stationary program $\bar{v}$ is a vector satisfying (8.1) for which $u(v)$ is a maximum.

2 To be completely correct here one must also assume that $u$ has a support at all points $v$. A counterexample in 1-dimension would be $u(v)=v^{1 / 2}$ which has no support at $v=0$. 
Mathematically, if $V$ is unbounded it might be that no optimal stationary program existed. However, for the present model, it is eminently reasonable that there should exist such a program. It corresponds to the economic millennium, or as economists have come to call it, the Golden Age, and it represents the steady state which would occur after we had built all the productive equipment which our resources could support on a steady basis. The theorems of this section will show that optimal stationary programs play a central role in the theory of optimal development. The starting point, as usual, will be the Kuhn-Tucker Theorem.

LEMma. If $\bar{v}$ is an optimal stationary program there exists an m-vector $p$ such that

$$
u(v)+p(B-A) v \text { is maximized at } \bar{v} \text { for all } v \text { in } V \text {. }
$$

The proof is a direct application of the Kuhn-Tucker Theorem.

It is convenient from now on to assume that $u(\bar{v})=0$, that is, that the maximum sustainable utility is zero. This can obviously be achieved by adding a suitable constant to $u$ if necessary. The first important result is

TheOREM 5. There exists a constant $M$ such that for any program $\left\langle v_{t}\right\rangle$ and any time $T, \sum_{t=1}^{T} u\left(v_{t}\right) \leqq M$.

Proof. For the program $\left\langle v_{t}\right\rangle$ we have from (8.2)

$$
u\left(v_{t}\right)+p(B-A) v_{t} \leqq 0 \quad \text { for all } t,
$$

so

$$
\sum_{t=0}^{T} u\left(v_{t}\right) \leqq \sum_{t=0}^{T} p(A-B) v_{t}=p A v_{0}-p B v_{T}=p s-p B v_{T} \leqq M,
$$

for since $v_{T}$ is bounded, so is $p B v_{T}$.

Theorem 5 shows that for all programs the partial sums $\sum_{t=0}^{T} u_{t}\left(v_{t}\right)$ are uniformly bounded above. That is, no program can be infinitely better than an optimal stationary program. They can, however, be infinitely worse, that is, $\sum_{t=0}^{T} u_{t}\left(v_{t}\right)$ can be unbounded below. This suggests

Definition. A program $\left\langle v_{t}\right\rangle$ is called good if the partial sums $\sum_{t=1}^{T} u\left(v_{t}\right)$ are bounded below.

We now obtain an asymptotic theorem for good programs.

THEOREM 6. If $u$ is strictly concave and the program $\left\langle v_{t}\right\rangle$ is good, then

$$
\begin{gathered}
\sum u\left(v_{t}\right) \text { converges, } \\
\lim _{t \rightarrow \infty} v_{t}=\bar{v} .
\end{gathered}
$$


(Note that since $u$ is strictly concave $\bar{v}$ is unique.)

Proof. From (8.3) we have

$$
u\left(v_{t}\right)=p(A-B) v_{t}-\delta_{t} \quad \text { where } \delta_{t} \geqq 0 .
$$

Now since $\left\langle v_{t}\right\rangle$ is good there exists $N$ such that

$$
N \leqq \sum_{t=0}^{T} u\left(v_{t}\right)=p s-p B v_{T}-\sum_{t=0}^{T} \delta_{t} \leqq M-\sum_{t=0}^{T} \delta_{t}
$$

or $\sum_{t=0}^{T} \delta_{t} \leqq M-N$ so $\sum_{t=0}^{\infty} \delta_{t}$ converges and in particular $\lim _{t \rightarrow \infty} \delta_{t}$ $=0$. This means from (8.6)

$$
\lim _{t \rightarrow \infty} u\left(v_{t}\right)+p(B-A) v_{t}=0
$$

but 0 is the maximum of $u(v)+p(B-A) v$ and it is achieved only at $\bar{v}$ since $u$ is strictly concave there, so $\lim _{t \rightarrow \infty} v_{t}=\bar{v}$ proving (8.5).

To prove (8.4) note from (8.7) that

$$
\sum_{t=0}^{T} u\left(v_{t}\right)=p s-p B v_{T}-\sum_{t=0}^{T} \delta_{t}
$$

and since $\nu_{T} \rightarrow \bar{v}$ and $\sum_{t=0}^{T} \delta_{t}$ converges, we have the desired convergence.

Now in looking for an optimal program it is clear that we need only consider good programs, hence we may look among the convergent infinite series $\sum_{t=0}^{\infty} u\left(v_{t}\right)$ for one with the largest sum. We have already seen that these sums are bounded in Theorem 5 so it is only a matter of showing that the supremum is achieved. This turns out not to be so easy. Here is a counterexample. Suppose we start with one unit of cake and each day we must decide how much to consume and how much to save. Clearly, a program is just a sequence $\left\langle c_{t}\right\rangle$ of consumptions subject to the condition $c_{t} \leqq 1$. Now if $u$ is any strictly concave function of $c$ then there can be no optimal program, for if $\left\langle c_{t}\right\rangle$ is a program then there must be some time $T$ such that $c_{r} \neq c_{T+1}$, but in this case $\left\langle c_{t}\right\rangle$ is overtaken by the program $\left\langle c_{t}^{\prime}\right\rangle$ where $c_{t}^{\prime}=c_{t}$ for $t \neq T, T+1$ and $c_{T}^{\prime}=c_{T+1}^{\prime}=\left(c_{T}+c_{T+1}\right) / 2$.

Despite the above example one can get a concise existence theorem for this model. Once again this requires the use of prices and competitive programs.

THEOREM 7. If $u$ is strictly concave then there exists a program $\left\langle v_{t}\right\rangle$ which is

(a) good 
(b) competitive

(c) the prices $p_{t}$ are bounded.

We will not prove this theorem which is a technical matter of obtaining $\left\langle v_{t}\right\rangle$ as a limit of optimal $T$-period programs. A somewhat delicate part is the verification of condition (c). The interested reader is referred to [1]. Assuming this theorem we get

TheOREM 8. If $u$ is strictly concave then the program $\left\langle v_{t}\right\rangle$ of Theorem 7 is strongly optimal.

Proof. Let $\left\langle v_{t}^{\prime}\right\rangle$ be any other good program. From (7.1)

$u\left(v_{t}^{\prime}\right)+\left(p_{t+1} B-p_{t} A\right) v_{t}^{\prime}+\delta_{t}=u\left(v_{t}\right)+\left(p_{t+1} B-p_{t} A\right) v_{t} \quad$ for all $t$

where $\delta_{t} \geqq 0$. Hence

or

$$
\sum_{t=0}^{T} u\left(v_{t}^{\prime}\right)+p_{T+1} B v_{T}^{\prime}-p_{0} s+\sum_{t=0}^{T} \delta_{t}=\sum u\left(v_{t}\right)+p_{T+1} B v_{T}-p_{0} s
$$

$$
\sum_{t=0}^{T}\left(u\left(v_{t}^{\prime}\right)-u\left(v_{t}\right)\right)=p_{T+1} B\left(v_{T}-v_{T}^{\prime}\right)-\sum_{t=0}^{T} \delta_{t} .
$$

Now if $v_{t} \neq v_{t}^{\prime}$ then by strict concavity $\delta_{t}>0$, hence the last term in (8.8) becomes negative. By Theorem 6 , the term $\left(v_{t}-v_{t}^{\prime}\right) \rightarrow 0$ since $\left\langle v_{t}\right\rangle$ and $\left\langle v_{t}^{\prime}\right\rangle$ are both good programs and since the $p_{t}$ are bounded, the term $p_{T+1} B\left(v_{T}-v_{T^{\prime}}\right) \rightarrow 0$. Hence, the right-hand side of (8.8) eventually becomes negative, so $\left\langle v_{t}\right\rangle$ overtakes $\left\langle v_{t}^{\prime}\right\rangle$.

(One might ask why the cake eating example does not fall under this theorem. The answer is that in that example $u$ fails to be strictly concave in the sense required. Notice that there are two activities. You can't eat your cake and have it too, but you can eat some of it and have the rest. If we had attached utility both to the eating and having in a strictly concave manner then there would have been an optimal program. As given the example fails to satisfy (8.5) of Theorem 7.)

COROLLARY. If $s=B \bar{v}$ then the program $\langle\bar{v}\rangle$ is strongly optimal.

Proof. The program $\langle\bar{v}, p\rangle$ clearly satisfies the conditions of Theorem 7 , hence is optimal by Theorem 8 .

The corollary says that the optimal stationary program is optimal. This is not a trivial tautology, for to say that $\langle\bar{v}\rangle$ is optimal stationary means that it is better than any other stationary program, but it is still conceivable that starting from $s=B \bar{v}$ there could be a nonstationary program better than $\langle\bar{v}\rangle$. 
9. The case of discounting. We shall give one more application of competitive prices, this time to show how they can be used to produce counterexamples to conjectures one might make. This work was done by W. R. Sutherland in his doctoral dissertation [5].

In the previous section it was assumed that $u$ was strictly concave and independent of time and we showed that all good programs approach a unique optimal stationary program. Now it is quite usual in development theory to consider discounted utilities, that is utility function $u_{t}$ where $u_{t}(v)=\delta^{t} u(v)$, and $\delta$ is some positive number less than unity. In this case if say $V$ is bounded then the existence of optimal programs becomes very easy since all utility series converge. On the other hand, as we will see, the qualitative behavior in relation to stationary programs may be quite complicated. In fact we shall give a simple model in which there are exactly two distinct stationary programs which are optimal. This is demonstrated by using prices in an essential way.

We consider a very simple model in which there is a single good. There are two activities, producing and consuming so that the $A$ and $B$ matrix become $(0,1)$ and $(1,0)$, respectively. Let us write the activity vector as $v=(l, c)$ where $l$ stands for the labor used for production and $c$ stands for consumption. In order for $\left\langle v_{t}\right\rangle$ to be a program the condition is simply

$$
c_{0}=s, \quad c_{t}=l_{t-1} .
$$

We may prescribe the set $V$ of pairs $(l, c)$ quite arbitrarily provided it is bounded. The corresponding utility requirement $\left\langle u_{t}\right\rangle$ is given by $u_{t}=\delta^{t} u\left(l_{t}, c_{t}\right)$ and a stationary program is then one for which $\left(l_{t}, c_{t}\right)$ $=(s, s)$ for all $t$.

TheOREM 9. A stationary program $\langle s, s\rangle$ is optimal if there is a price $p$ such that $u(l, c)+\delta p l-p c$ is maximized at $(s, s)$.

Proof. Let $\left\langle l_{t}, c_{t}\right\rangle$ be any program from $s$. Then

$$
u\left(l_{t}, c_{t}\right)+\delta p l_{t}-p c_{t} \leqq u(s, s)+\delta p s-p s .
$$

Multiplying by $\delta^{t}$ and summing, using $c_{t}=l_{t-1}$, gives

$$
\begin{aligned}
\sum_{t=0}^{T} \delta^{t}\left(u\left(l_{t}, c_{t}\right)-u(s, s)\right) & \leqq\left(\delta^{T+1}-1\right) p s-\left(\delta^{T+1} p l_{T}-p c_{0}\right) \\
& =\delta^{T+1} p\left(s-l_{T}\right)
\end{aligned}
$$

and since the right-hand side approaches zero, the optimality is proved. 
The converse of this theorem is true under suitable convexity assumptions but we will not need it.

COROLLARY. If $u$ is strictly concave and

$$
(\partial u / \partial l)(s, s)=-\delta(\partial u / \partial c)(s, s) .
$$

Then $\langle s, s\rangle$ is optimal. Then

PROOF. Let $p=(\partial u / \partial c)(s, s)$ and let $\phi(l, c)=u(l, c)+\delta p l-p c$.

$$
(\partial \phi / \partial l)(s, s)=(\partial u / \partial l)(s, s)+\delta p=(\partial u / \partial l)(s, s)+\delta(\partial u / \partial c)(s, s)=0
$$

from (9.1), and $(\partial \phi / \partial c)(s, s)=(\partial u / \partial c)(s, s)-p=0$ by definition of $p$. Hence $(s, s)$ is a critical point of $\phi$ but since $\phi$ is strictly concave, $(s, s)$ must maximize $\phi$ and the conclusion follows from the theorem.

To prove nonuniqueness it suffices to find a strictly concave function $u(l, c)$ for which the conditions of the corollary hold at two distinct points. Such a function turns out to be

$$
u(l, c)=21, \log (3.4+1.1 c-l)+\log (4+2 c-l)+\frac{3}{4} c .
$$

Note that besides being strictly concave, $u$ is increasing in $c$ and decreasing in $l$ reflecting the natural condition that consuming is pleasant and producing is unpleasant. We leave it to the enterprising reader to verify that condition $(9.1)$ is satisfied both at $(1,1)$ and $(2,2)$.

Obviously, if there is more than one stationary program which is optimal, then one cannot expect asymptotic theorems like Theorem 1. In fact Sutherland has given an example in which although there is a unique optimal stationary program $\langle(s, s)\rangle$, any program starting from stocks $s^{\prime}$ different from $s$ moves away from this program and approaches some oscillating solution in which one produces on the odd days and consumes on the even.

\section{BIBLIOGRAPHY}

1. D. Gale, On optimal development in a multi-sector economy, Rev. Econ. Studies 34 (1967), 1-18.

2. D. Gale and W. R. Sutherland, Analysis of a one good model of economic development, Proc. Summer Seminar on Math. Decision Sciences, Amer. Math. Soc. (to appear).

3. Daniel McFadden, The evaluation of development programmes, Rev. of Econ. Studies 34 (1967), 25-50.

4. J. von Neumann, On a system of equations in economics and a generalization of the Brouwer fixed point theorem, Rev. Econ. Studies, 1945-46.

5. W. R. Sutherland, On optimal development programs when future utility is discounted, Doctoral Dissertation, Brown University, Providence, R.I., 1967.

University of California, Berkeley 